\title{
EVOLUÇÃO CLÍNICA DA SÍNDROME SARS-COV-2 EM PACIENTE PORTADOR DE HIPERTENSÃO, DIABETES TIPO II E SÍNDROME METABÓLICA
}

\section{CLINICAL EVOLUTION OF SARS-COV-2 SYNDROME IN A PATIENT WITH HYPERTENSION, TYPE II DIABETES AND METABOLIC SYNDROME}

\author{
Renata Souza Poubel de Paula1, Marina Hübner Freitas dos Santos Silva Machado², Bruno Beber Machado², \\ Augusto Paiva Moraes ${ }^{4}$, Roberto Batista Marques Junior ${ }^{5}$ \\ ${ }^{1}$ Mestra em Planejamento Regional e Gestão de Cidades - UCAM - Brasil; Acadêmica de Medicina - \\ Universidade Iguaçu - Campus V - UNIG - Brasil; \\ 2 Acadêmica de Medicina - Universidade Iguaçu - Campus V - UNIG - Brasil; \\ ${ }^{3}$ Médico Radiologista - Universidade Federal Fluminense - UFF- Brasil; Mestre em Radiologia e Diagnóstico por \\ Imagem - Universidade Federal do Rio de Janeiro - UFRJ- Brasil; Membro do Colégio Brasileiro de Radiologia \\ (CBR). \\ ${ }^{4}$ Acadêmico de Medicina - Universidade Iguaçu - Campus V- UNIG - Brasil; \\ ${ }^{5}$ Doutor em Produção Vegetal - UENF- Brasil; Acadêmico em Medicina - Universidade Iguaçu - Campus V - \\ UNIG - Brasil.
}

Nome do Autor de Correspondência: Renata Souza Poubel de Paula

Endereço: Rua Coronel Raul Costa

Telefone: (22) 988267593

E-mail de correspondência: renatapoubel85@gmail.com

RESUMO

A pandemia da COVID-19 trouxe uma corrida em busca da cura para as infecções pelo SARS-CoV-2. Os sintomas mais relatados são febre, tosse, dispneia e dificuldades respiratórias, embora muitos casos sejam declarados assintomáticos. Há um grande investimento na ciência e tecnologia nesse momento e, também, muitos questionamentos a respeito da ética na condução dessas pesquisas. Objetivo: 0 objetivo desse estudo é descrever a evolução da síndrome ocasionada pelo SARS-CoV-2 no paciente e 'as medidas adotadas durante o seu tratamento. Metodologia: O método utilizado foi um relato de caso de um paciente diagnosticado com COVID-19, portador de hipertensão, diabetes tipo II e síndrome metabólica, com comprometimento pulmonar significativo e infiltrado em vidro fosco difuso, que evoluiu para internação na unidade de terapia intensiva - UTI. O protocolo de posição prona, preconizado para o tratamento de pacientes acometidos pelo COVID-19, não pode ser utilizado durante a internação, devido a sua obesidade. Resultados: O paciente apresentou hipoxemia associada a dispneia, com episódios de dessaturação,

\section{ABASTRACT}

The COVID-19 pandemic has brought a race to find a cure for SARS-CoV-2 infections. The most reported symptoms are fever, cough, dyspnoea and breathing difficulties, although many cases have been declared asymptomatic. There is a great investment in science and technology at this moment and, also, many questions about ethics in conducting these researches. Objective: The objective of this study is to describe the evolution of SARS-CoV-2 syndrome in the patient and the measures taken during his treatment. Methodology: The method used was a case report of a patient diagnosed with Covid-19, with hypertension, type II diabetes and metabolic syndrome, with significant pulmonary impairment and diffuse groundglass infiltration, who progressed to hospitalization in the intensive care unit - ICU. The prone position protocol, recommended for the treatment of patients affected by COVID-19, can not be used during hospitalization, due to its obesity. Results: The patient presented hypoxemia associated with dyspnea, with episodes of desaturation, with the need for oxygen therapy and non-invasive ventilation on a constant basis, using an $\mathrm{O} 2$ nasal cannula. Conclusion: The prone 
com a necessidade de oxigenoterapia e ventilação não invasiva de forma constante, por meio de cânula nasal de O2. Conclusão: A posição prona é segura e deve ser utilizada sempre que possível por favorecer a resposta positiva ao tratamento, entretanto, o paciente recuperou-se do quadro sem realizá-la. É fundamental a monitorização do paciente, assim como acompanhar a evolução pulmonar da doença, por meio de exames de imagem.

Palavras-chave: COVID-19; vidro fosco; obesidade; ventilação não invasiva.

\section{INTRODUÇÃO}

Cientistas e pesquisadores estão se mobilizando no mundo inteiro, desde a chegada da pandemia do COVID-19, em busca de desenvolverem uma vacina contra o vírus, utilizando-se de grandes investimentos na área da ciência e tecnologia, desenvolvendo estudos clínicos1 para a utilização dos medicamentos já existentes e até mesmo para a produção de novos fármacos. Foram muitos questionamentos a respeito da utilização da hidroxicloroquina1 e a sua combinação com outros fármacos, como ivermectina, azitromicina, zinco e vitamina D. É de conhecimento científico que as pesquisas devem seguir boas práticas clínicas, considerando os princípios éticos de suma importância, baseados, principalmente, na Declaração de Helsinque3, que normalmente serve de base para a condução dos ensaios clínicos. Alguns fármacos como a Hydroxicloroquina e Cloroquina 2 têm sido testados em humanos, pois é uma medicação já usada no combate a outras doenças, como a malária e lúpus, e apresentaram ação in vitro positiva contra o vírus SARS-CoV-2. O paciente em questão fez uso dessa combinação, mas ocorreu um agravamento do seu quadro clínico, evoluindo para internação na UTI, com infiltrado em vidro fosco difuso nos pulmões. É importante entender a evolução desse paciente infectado, considerando suas particularidades e medidas adotadas durante o seu tratamento. Apresenta-se a seguir o relato de caso de um paciente infectado pelo COVID-19, portador de hipertensão, diabetes tipo Il e síndrome metabólica, e sua evolução, que culminou no agravamento do seu estado clínico geral e encaminhamento para a UTI.

\section{DESCRIÇÃO DO CASO}

Paciente do sexo masculino, 68 anos, position is safe and should be used whenever possible because it favors a positive response to treatment, however, the patient recovered from the condition without performing it. It is essential to monitor the patient, as well as monitor the pulmonary evolution of the disease, using imaging tests.

Keywords: COVID-19; frosted glass; obesity; noninvasive ventilation.

médico, dislipidêmico, obeso, hipertenso, diabético tipo II, não tabagista, etilista, portador de síndrome metabólica, sem histórico familiar de comorbidades em uso contínuo de hidroclorotiazida de 12,5, atenolol de $50 \mathrm{mg}$, losartana, glimepirida, metformina, dulaglutida. Relata ter sido infectado em uma pequena comemoração familiar no dia 07 de junho. Os primeiros sintomas surgiram 5 dias depois, quando foi diagnosticada a doença por meio da Reação em Cadeia da Polimerase em Tempo Real (RT-PCR). Com a evolução do quadro e dificuldades respiratórias associadas, buscou auxílio médico, dando entrada no hospital no dia 14 de junho ("Fig. 1 1"), quando foi submetido a exames radiográfico e tomográfico, iniciando sua internação. Com o agravamento de sua função respiratória e manutenção da saturação sempre ao redor de $88 \%$ dessaturação constante, o paciente foi transferido do quarto para a UTI 8 dias após a sua internação, devido a necessidade de oxigenoterapia e ventilação não invasiva 8, de forma constante, através de cânula nasal de 02 . O quadro clínico se agravou, com piora da dispneia, tosse, febre, sudorese. Ao exame físico, evoluía com diminuição de murmúrio vesicular bilateralmente. Após 5 dias de internação na UTI, foi transferido para o quarto, permanecendo por mais 4 dias no hospital até receber alta.

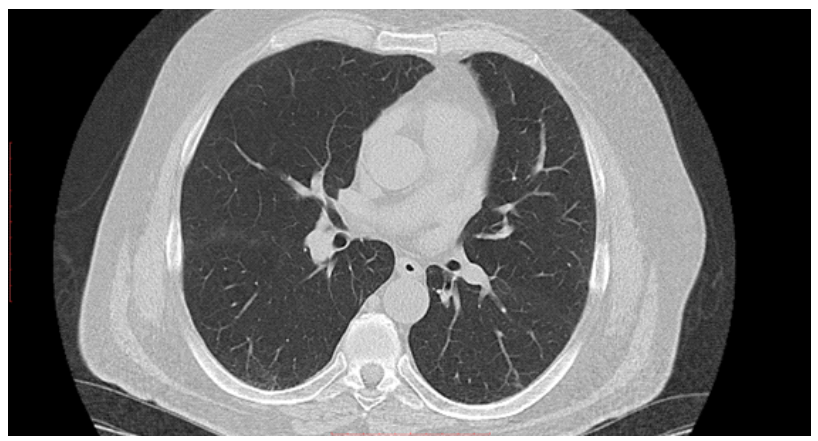

FIGURA 1: TC de tórax 14.06 - Discreto infiltrado em vidro foscocomprometimento pulmonar menor que $5 \%$ 


\section{DISCUSSÃO}

$\mathrm{O}$ relato de caso mostra que o paciente, inicialmente, apresentou dor, febre, calafrios, sudorese, dispneia e dessaturação8, sintomas comuns ao COVID-19, como febre, tosse e mialgia ou fadiga e menos frequente a produção de escarro, dor de cabeça, hemoptise e diarreia, sendo que mais da metade dos pacientes desenvolvem dispneia. Pacientes hipoximêmicos possuem um aumento da frequência respiratória como resposta fisiológica a diminuição da oxigenação no sangue6. Além disso, o paciente possui algumas comorbidades que influenciam na potencialidade de agravamento do seu estado4. No RX de tórax, realizado em 14 de junho, o paciente apresentou um discreto infiltrado em vidro-fosco, com comprometimento pulmonar menor que 5\%. Já em 22 de junho ("Fig. 2"), foi
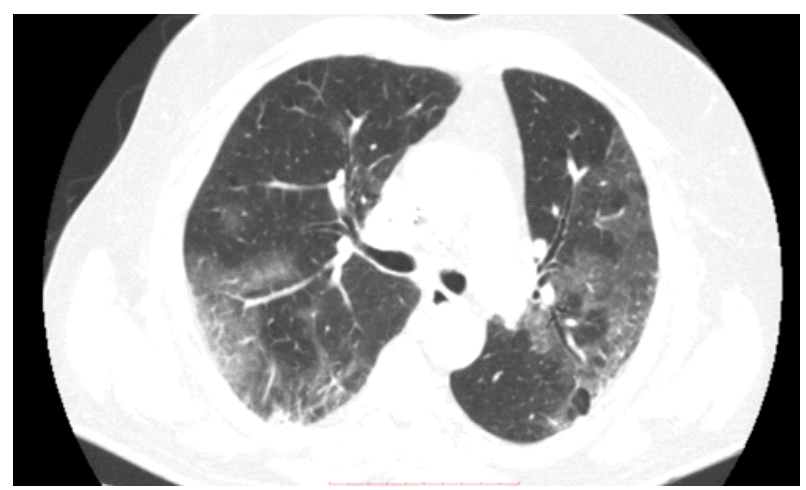

Figura 2: TC DE TÓRAX 22.06: Aumento para mais de $50 \%$ do comprometimento pulmonar- Paciente internado, com piora do quadro radiológico e clínico, mas ainda fora do UTI. Após esse exame, o paciente foi transferido para o UTI

realizada uma tomografia computadorizada de tórax, onde pode ser constatado um aumento para mais de $50 \%$ de comprometimento pulmonar. 0 paciente permanecia internado, com piora do quadro clínico atestado pelos exames complementares, mas ainda fora da UTI. Nesse mesmo dia, após as constantes dessaturações do paciente, chegando a $83 \%$, ele foi transferido para a UTI, a fim de realizar-se um acompanhamento clínico minucioso, sob a forte possibilidade de intubação. No dia 23 de junho ("Fig. 3") foi realizado outro RX de tórax, agora no leito, mostrando pulmões pouco expandidos, com infiltrado em vidro fosco difuso. A maioria dos casos publicados atualmente possui achados tomográficos semelhantes, como opacidades em vidro fosco7. 0 paciente estava em uso de ventilação não invasiva (VNI) e com po2 baixa. No dia 24 o quadro se

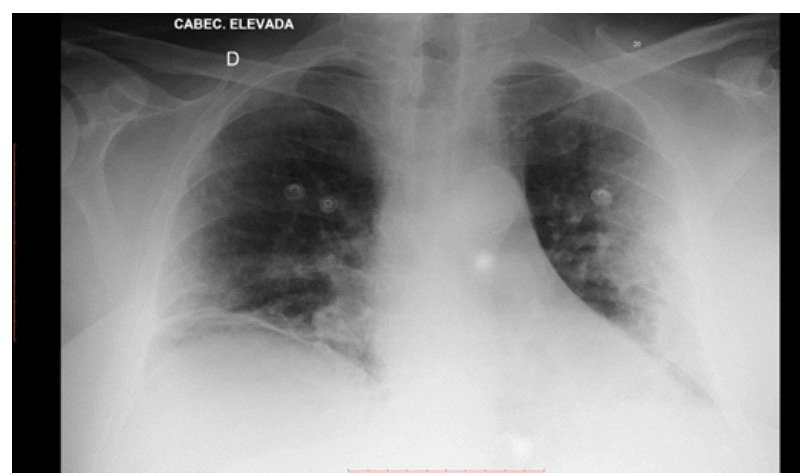

Figura 3: RX DE TÓRAX 23.06- Exame realizado no leito, em UTI, mostra pulmões pouco expandidos, com infiltrado em vidro fosco difuso. O paciente está em VNI (ventilação não invasiva) e com po2 baixa.

mantinha igual, com piora clínica e radiológica ("Fig. $4 ")$. Dois dias depois, dia 26, o paciente ainda apresentava o mesmo comprometimento

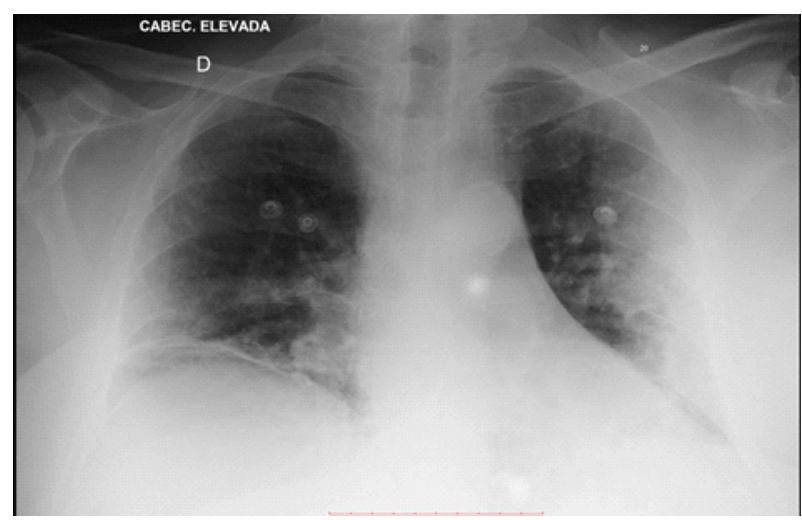

Figura 4: RX DE TÓRAX 24.06- Exame realizado no leito, em UTI, mostra pulmões pouco expandidos, com infiltrado em vidro fosco difuso. O paciente está em VNI (ventilação não invasiva) e com po2 baixa. $O$ paciente apresenta piora clínica e radiológica.

pulmonar, porém com estabilidade clínica e radiológica ("Fig. 5"). A obesidade é um dos fatores

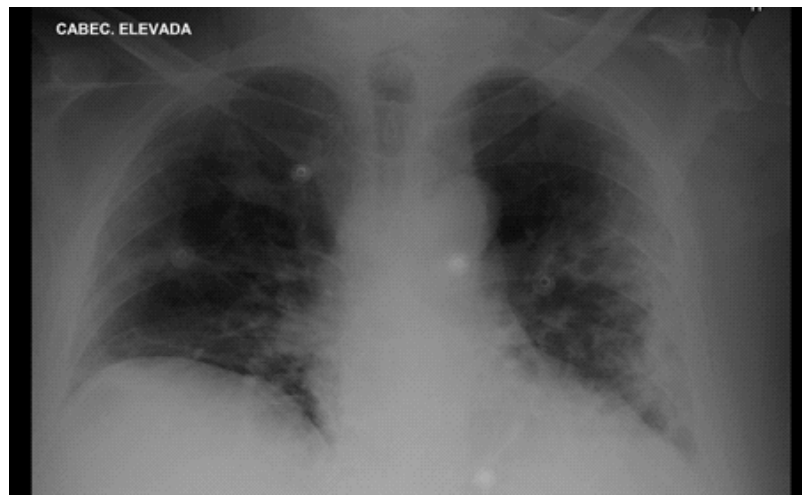

Figura 5: RX DE TÓRAX 26.06- Exame realizado no leito, em UTI, mostra pulmões pouco expandidos, com infiltrado em vidro fosco difuso. $O$ paciente está em VNI (ventilação não invasiva) e com po2 baixa. 0 paciente apresenta estabilidade clínica e radiológica. 
de risco para a evolução com piora desse quadro5. Entender os mecanismos fisiopatológicos dessa relação entre obesidade e agravamento da COVID19 é fundamental. O paciente começou a recuperarse, gradualmente, até a obtenção da alta hospitalar. Durante o tratamento foi utilizado ceftriaxona, corticoide, broncodilatador e fisioterapia respiratória. Novas pesquisas que relacionem a morbimortalidade da obesidade e o COVID-19 são fundamentais para que o estímulo de vida saudável possa ser cada vez mais incentivado5.

\section{CONCLUSÃO}

A COVID-19 determinou uma situação emergencial que permitiu a diversos pesquisadores iniciarem ensaios clínicos, a fim de alcançarem uma direção para mitigar os problemas trazidos junto à epidemia. $O$ esforço da equipe médica em realizar um planejamento em sua condução e um monitoramento do paciente, seguindo protocolos e ajustando de acordo com a evolução de cada caso, possibilitou mais um caso de recuperação como o do paciente em análise. A responsabilidade dos profissionais de saúde é enorme assim como a coletiva em evitar a contaminação. Portanto, há uma necessidade de discutir sobre a problemática, observando a experiência clínica e o desenvolvimento da doença.

\section{REFERÊNCIAS}

1- Brasil. Ministério da Saúde. Departamento de Ciência e Tecnologia. Secretaria de Ciência, Tecnologia, Inovação e Insumos Estratégicos. Informe diário de evidências COVID-19. Busca realiza entre os dias 18 e 20 de setembro (internet) 2020. [acesso 20 de set 2020]. Disponível em http://saude.gov.br/images/pdf/2020/September/02/N103InformeDiario-referente-28-08.pdf

02- Borba, MGS, Val FA, Sampaio VS, Alexandre MAA, Melo GC, Brito M et al. Cloroquine diphosphate in two diferente dosages as adjunctive therapy of hospitalized patients with severe respiratory syndrome in the contexto o coronavirus (SARS - COV-2) infection: Preliminary safety results of a randomized, double-blinded, phase Ilb clinical trial (CloroCovid-19 Study). 2020. [acesso 20 set 2020]. Disponível em: https://www.medrxiv.org/content/10.1101/2020.04.07.20056424v2

3- World Medical Association. Declaração de Helsinque. Princípios éticos para a pesquisa médica envolvendo seres humanos [internet] [acesso em 21 set. 2020]. Disponível em: https://arquivos.amb.org.br/downloads/ 491535001395167888_DoHBrazilianPortugueseVersionRev.pdf 4- NIQUINI, Roberta Pereira et al. SRAG por COVID-19 no Brasil: descrição e comparação de características demográficas e comorbidades com SRAG por influenza e com a população geral. Cadernos de Saúde Pública, v. 36, p. e00149420, 2020.[acesso em 19 set. 2020] Disponível em: https://www.scielosp.org/article/csp/2020.v36n7/e00149420/pt/

5- MOREIRA, Gláucia Sabino; REIS, Lílian Barros de Sousa Moreira; FREIRE, Patrícia Barbosa. Obesidade e agravamento da COVID-19. Health Residencies Journal-HRJ, v. 1, n. 6, p. 63-70, 2020. [acesso em 15 set. 2020] Disponível em https://escsresidencias.emnuvens .com.br/hrj/article/view/27/59 6- DOS ANJOS, Jorge Luis Motta et al. Posição prona em pacientes em ventilação espontânea com insuficiência respiratória por COVID-19: relato de caso. Revista Pesquisa em Fisioterapia, v. 10, n. 3, 2020.

7- ARAUJO-FILHO, Jose de Arimateia Batista et al. Pneumonia por COVID-19: qual o papel da imagem no diagnóstico?. Jornal Brasileiro de Pneumologia, v. 46, n. 2, 2020.

8- MARTINEZ, Bruno Prata et al. Indicação e uso da ventilação não-invasiva e da cânula nasal de alto fluxo, e orientações sobre manejo da ventilação mecânica invasiva no tratamento da insuficiência respiratória aguda na COVID-19. ASSOBRAFIR Ciência, v. 11, n. Supl1, p. 101-110. 